\title{
Intraoperative magnetic resonance imaging-augmented transoral resection of axial disease
}

\author{
Taro Kaibara, M.D., R. John Hurlbert, M.D., and Garnette R. Sutherland, M.D. \\ The Seaman Family MR Research Center, Division of Neurosurgery, Department of Clinical \\ Neurosciences, University of Calgary, Alberta, Canada
}

\begin{abstract}
Object. Because transoral decompression of the cervicomedullary junction is compromised by a narrow surgical corridor, the adequacy of decompression/resection may be difficult to determine. This is problematic as spinal hardware may obscure postoperative radiological assessment, or the patient may require reoperation. The authors report three patients in whom high-field intraoperative magnetic resonance (MR) images were acquired at various stages during the transoral resection of $\mathrm{C}-2$ lesions causing craniocervical junction compression.

Methods. In all three patients the lesions involved the cervicomedullary junction: one case each of plasmacytoma and metastatic breast carcinoma involving the odontoid process and C-2 vertebral body, and one case of basilar invagination with a Chiari type I malformation. All three patients presented with progressive myelopathy. Surgery-planning MR imaging studies, performed after the induction of anesthesia, demonstrated the lesion and its relationship to the planned surgical corridor. Transoral exposure was achieved through placement of a Crockard retractor system. In one case the soft palate was divided. Interdissection MR imaging revealed that adequate decompression had been achieved in all cases. In the two patients with carcinoma, posterior instrumentation was placed to achieve spinal stabilization. Planned suboccipital decompression and fixation was averted in the third case because MR imaging demonstrated that excellent decompression had been achieved.

Conclusions. Intraoperatively acquired MR images were instrumental in determining the adequacy of surgical decompression. In one patient the MR images changed the planned surgical procedure. Importantly, the acquisition of intraoperative MR images did not adversely affect operative time or neurosurgical techniques, including the instrumentation procedure.
\end{abstract}

\section{KEY WORDS - intraoperative monitoring - magnetic resonance imaging - transoral approach • cervical spine • cervicomedullary junction}

The surgical treatment of ventral upper cervical spine and skull base disease remains challenging because of their anatomical complexity and limited surgical accessibility. The transoral pathway provides a direct and useful approach to lesions involving the craniocervical junction. The transoral approach has been described in the surgical treatment of a variety of extra- and intradural lesions, ${ }^{1,3-7}$, 11-14,18-23 including rheumatoid disease, glomus tumors, chordomas, meningiomas, schwannomas, platybasia, fractures, and metastasis; its popularity has grown with improved understanding of the biomechanics of the craniovertebral anatomy. ${ }^{8,9}$ However, because of the inherently deep and narrow surgical corridor, which is further restricted by the essential use of retractors, the extent of resection and medullary decompression may be difficult to visualize intraoperatively.,5

Intraoperative MR imaging systems have been available for several years. ${ }^{2,15,24-26}$ However, their application in the spine has been limited. A high-field mobile MR imaging system has been developed so that neurosurgeons may

Abbreviations used in this paper: $\mathrm{MR}=$ magnetic resonance; $\mathrm{RF}=$ radiofrequency. guide and observe the effects of resection on the central nervous system and associated structures. ${ }^{16,17,25}$ The system, described in detail elsewhere, ${ }^{16,17,25}$ is based on an actively shielded 1.5 tesla, ceiling-mounted magnet that can be moved into and out of the operating room, as needed, throughout a given procedure. The system includes an RF coil, which can be disassembled, and local RF shielding. Because the magnetic fringe fields do not affect the operative field during surgery, all standard operating tools, instruments, and techniques can be used. This system was used to monitor the resection in three patients with $\mathrm{C}-2$ vertebral lesions causing extradural compression.

\section{CLINICAL MATERIAL AND METHODS}

Three patients presented with neck pain and myelopathy secondary to ventral extradural compression at the C1-2 level. Two patients, one 51 and the other 81 years of age, harbored metastatic lesions from metastatic breast carcinoma and plasmacytoma, respectively. These lesions resulted in the infiltration and destruction of the C-2 vertebral body and odontoid process that subsequently caused ventral cord compression. The third case, a 63- 
year-old patient, suffered from degenerative basilar invagination and a Chiari I malformation causing marked spinal canal narrowing and medullary compression.

All three patients underwent surgery via a transoral approach for decompression of the ventral C1-2 complex. A Crockard retractor system as well as other standard otolaryngological and neurosurgical instruments were used, including a high-speed air drill and an operating microscope. In the patient with basilar invagination soft palatectomy was performed to obtain adequate access.

\section{RESULTS}

\section{Intraoperative MR System and Imaging}

The MR imaging system is based on a 1.5-tesla actively shielded magnet suspended from the ceiling. The magnet is housed in a small alcove next to the operating theater and is moved into and out of the operating room by a small electric motor as required during an operation. Because the magnet is outside of the surgical field during dissection, all standard neurosurgical instruments and equipment can be used. During image acquisition all retractor systems and non-MR imaging-compatible equipment and instruments are removed from the surgical field.

Intraoperative images were acquired at various stages throughout the procedure. Surgery-planning images were acquired after the induction of anesthesia but prior to making an incision; interdissection images were obtained at any time during surgery by placing sterile drapes over the surgical site and moving the magnet into place over the patient; quality-assurance imaging was performed after wound closure but prior to reversal of anesthesia. Imaging time was approximately 2.5 minutes and 4.5 minutes for $\mathrm{T}_{1}$ - and $\mathrm{T}_{2}$-weighted sequences, respectively. Details of the MR imaging sequences and parameters have been described elsewhere. ${ }^{16,17,25}$

\section{Surgery and Intraoperative MR Imaging}

In the three patients a total of three surgery-planning, three interdissection, and one quality-assurance MR imaging studies were performed. These were comprised of seven $\mathrm{T}_{1}$-weighted images, four $\mathrm{T}_{2}$-weighted images, and one MR angiogram.

In the two patients with metastatic lesions of the C-2 body, microsurgical resection was performed via the transoral approach. Postresection, with the wound open, the retractors were removed, and interdissection images were obtained. These intraoperative MR images confirmed the gross-total resection of the tumor, excellent decompression of the spinal cord, and the absence of acute complications such as hemorrhage. Following intraoperative imaging, both patients were turned prone, immobilized with skull pins, and posterior occipitocervical fusion was performed. In the patient with metastatic breast disease a Mayfield loop (Ohio Medical Instrument Co., Cincinnati, $\mathrm{OH}$ ) was screwed to the occiput and fixed to the cervical spine $(\mathrm{C} 1-4)$ by sublaminar cables. In the patient with plasmacytoma, stabilization was achieved using an occipital Mayfield loop connected to C1-2 transarticular screws and C-3 and C-4 lateral mass screws. Autologous iliac crest graft was placed in both cases. Poststabilization MR imaging was not performed due to MR artifact from the hardware.

In the third patient, preoperative surgical planning included a transoral resection of the degenerative odontoidC1 complex and a posterior fossa decompression augmented by occipitocervical instrumentation. However, interdissection images revealed the medulla to be well decompressed, and there was good cerebrospinal fluid signal around the brainstem and upper cervical cord (Figs. 1 and 2). Following decompression, MR angiography demonstrated well the vertebral arteries, their relationship to the surgical corridor, and the impression of improved flow, increased signal. Based on inspection of the inter-

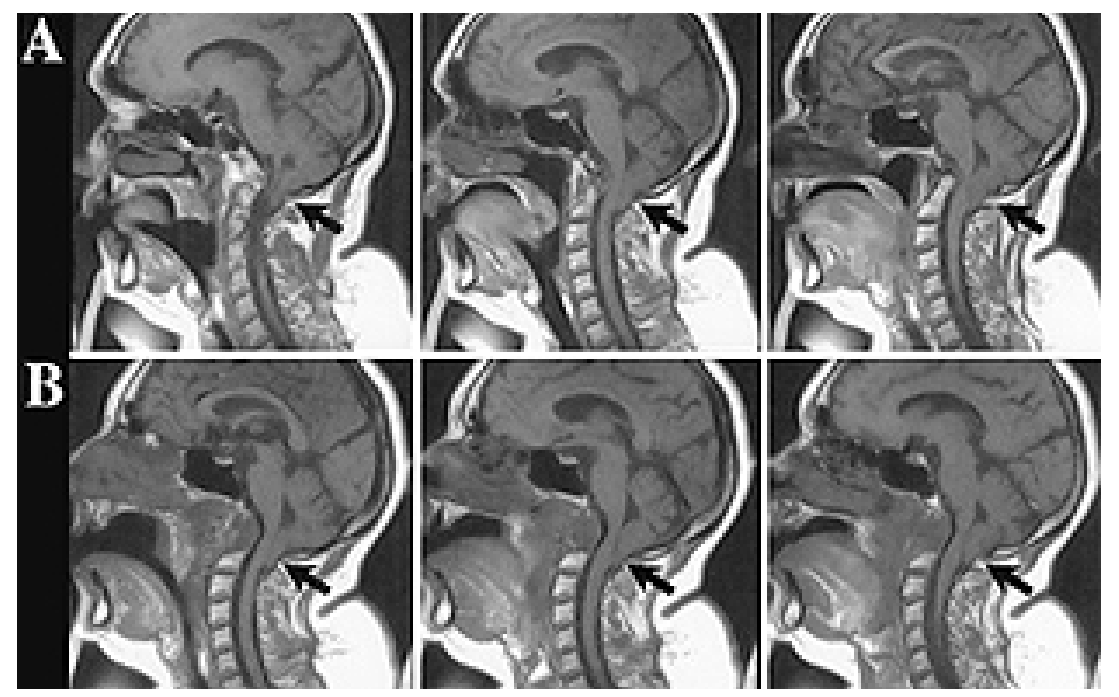

Fig. 1. Intraoperative sagittal $\mathrm{T}_{1}$-weighted MR images obtained in the patient with platybasia and Chiari I malformation. A: Surgery-planning images demonstrating impaction of the brainstem, odontoid process, and cerebellar tonsils in the foramen magnum (arrows). B: Interdissection images obtained following transoral resection of the odontoid-C1 complex, revealing decompression of the brainstem (arrows). 


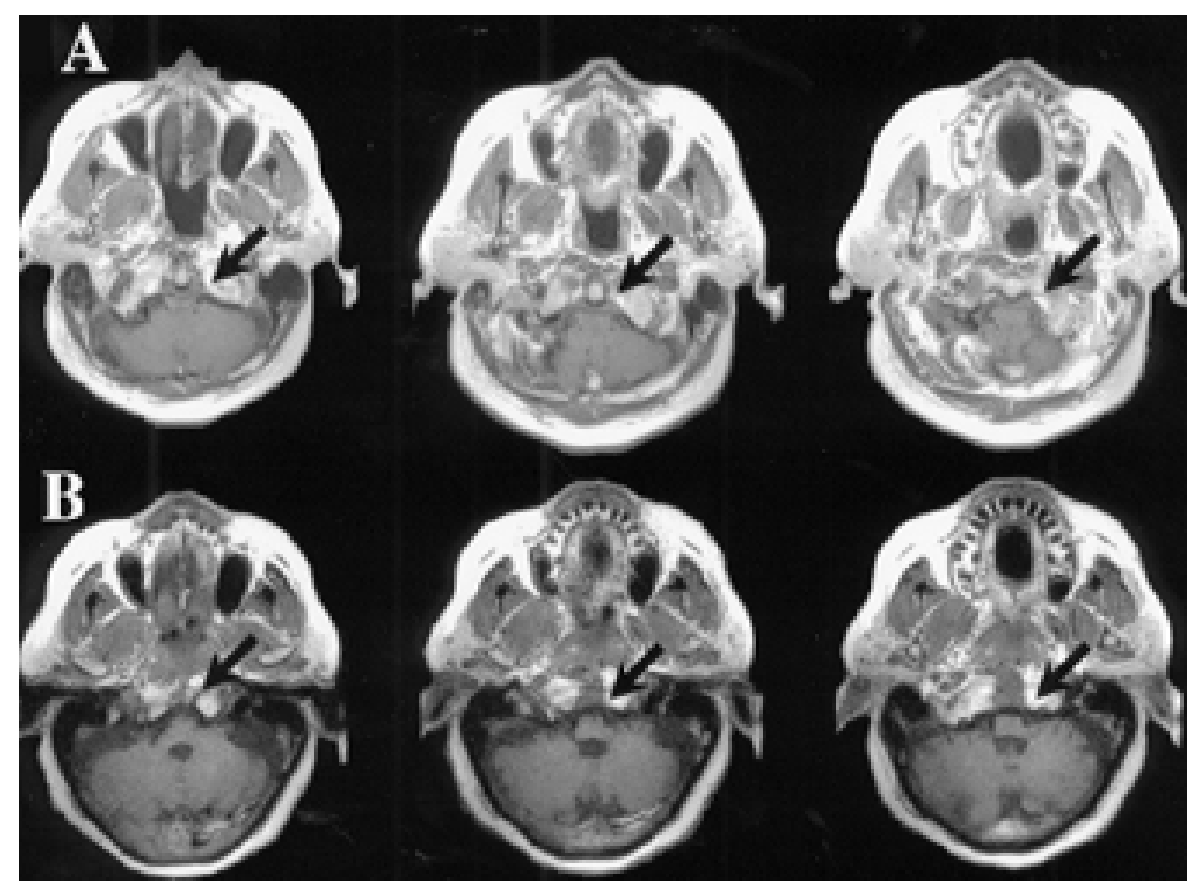

Fig. 2. Intraoperative axial $\mathrm{T}_{1}$-weighted $\mathrm{MR}$ images of the patient in Fig. 1. A: Surgery-planning images revealing significant ventral brainstem compression and deformation (arrows). B: Interdissection images obtained following transoral resection of the odontoid-C1 complex, demonstrating that the brainstem has been well decompressed (arrows).

dissection images, the planned posterior fossa decompression and fusion was not performed, and the patient was awakened from anesthesia.

Postoperatively, the patient with metastatic breast cancer and the patient with platybasia with Chiari malformation were well, with significant improvement of myelopathy at 9 months and 6 months, respectively. The patient with plasmacytoma died of renal failure shortly after surgery.

\section{DISCUSSION}

The mobile high-field MR system has been used to monitor and guide surgery in 180 patients with a broad spectrum of intracranial and cervical spine disease. . $^{16,17,25}$ At our institution the system has been successfully used in three patients undergoing transoral procedures to relieve cervicomedullary extradural compression caused by ventral C1-2 disease. Despite the use of an MR system, the use of complex spinal instrumentation was not adversely affected during these operations.

\section{Technological Features}

High-resolution MR images have been obtained in multiple plains of the cranial base and upper cervical spine. Such definition is not possible when using computerized tomography or lower-field MR systems. The RF coil design used in this study, with rings positioned above and below the patient's head, produces a large field of view with a uniformity not obtainable in the surface coil technology used by other investigators. ${ }^{2,15,24,26}$ High image resolution and field homogeneity are essential for visualizing well the effects of such procedures as resection of small lesions and neural decompression.

\section{Clinical Utility}

Although intraoperative imaging increases the operative time by approximately 30 minutes for each study, it proved invaluable at all surgical stages. Surgery-planning studies demonstrated the relationship of the lesion and patient positioning to the planned surgical corridor. They also updated diagnostic studies that had been obtained weeks prior to surgery. Interdissection studies demonstrated that the objective of surgery had been accomplished prior to placement of instrumentation. These data are particularly important because the provision of hardware introduces artifacts that render the interpretation of postoperative MR or computerized tomography images difficult. As was demonstrated in one case, knowledge that the cervicomedullary junction has been decompressed may obviate the need for other surgical interventions. In the future patients will undergo MR imaging evaluation once placed in the prone position for placement of posterior instrumentation. This will allow us to assess the effect of repositioning on the decompression and anatomical alignment of the spinal structures.

There have been a limited number of reports on the use of intraoperative MR imaging in spinal procedures. Hall, et al. ${ }^{15}$ have reported their experience with a few simple laminectomies for lumbar disc herniation and cervicothoracic syringosubarachnoid shunt placements. Specific requirements in patient positioning and specialized ferrous tools and equipment fundamental to instrumentation restrict the use of many intraoperative MR imaging systems in complex spinal procedures. Because the MR imaging system described herein is moved out of the surgical field during dissection, there are no restrictions on the types of surgical instruments or equipment used dur- 
ing the procedure, and all standard tools required to perform transoral surgery as well as complex spinal fixation can be used without adverse effects.

Intraoperative MR imaging technology is now married to surgical neuronavigation systems. A major shortcoming of neuronavigation systems, in which surgery is traditionally based on preoperatively acquired imaging data, is related to significant inaccuracies that result from intraoperative tissue deformation and shift. ${ }^{10,27}$ Intraoperatively acquired MR images used to update these systems are of significant potential value in improving their accuracy and thus utility. ${ }^{10,27}$ Furthermore, fixation of the spine that is guided by intraoperatively acquired neuronavigational data may eliminate the need for intraoperative fluoroscopy and the risks inherent in ionizing radiation.

The ability to evaluate the effect and extent of resection intraoperatively remains a tremendous advantage. This is particularly relevant in patients with ventral craniovertebral lesions in whom access and direct visualization via a transoral approach may otherwise be suboptimal.

\section{References}

1. Apuzzo ML, Weiss MH, Heiden JS: Transoral exposure of the atlantoaxial region. Neurosurgery 3:201-207, 1978

2. Black PM, Moriarty T, Alexander E III, et al: Development and implementation of intraoperative magnetic resonance imaging and its neurosurgical applications. Neurosurgery 41:831-842, 1997

3. Crockard HA: Transoral surgery: some lessons learned. Br J Neurosurg 9:283-293, 1995

4. Crockard HA, Bradford R: Transoral transclival removal of a schwannoma anterior to the craniocervical junction. Case report. J Neurosurg 62:293-295, 1985

5. Crockard HA, Sen CN: The transoral approach for the management of intradural lesions at the craniovertebral junction: review of 7 cases. Neurosurgery 28:88-97, 1991

6. Delgado TE, Garrido E, Harwick RD: Labiomandibular, transoral approach to chordomas in the clivus and upper cervical spine. Neurosurgery 8:675-679, 1981

7. Di Lorenzo N: Transoral approach to extradural lesions of the lower clivus and upper cervical spine: an experience of 19 cases. Neurosurgery 24:37-42, 1989

8. Dickman CA, Crawford NR, Brantley AG, et al: Biomechanical effects of transoral odontoidectomy. Neurosurgery 36: 1146-1152, 1995

9. Dickman CA, Locantro J, Fessler RG: The influence of transoral odontoid resection on stability of the craniovertebral junction. J Neurosurg 77:525-530, 1992

10. Dorward NL, Alberti O, Velani B, et al: Postimaging brain distortion: magnitude, correlates, and impact on neuronavigation. J Neurosurg 88:656-662, 1998

11. Estridge MN, Smith RA: Transoral fusion of odontoid fracture. Case report. J Neurosurg 27:462-465, 1967

12. Goel A, Bhatjiwale M, Desai K: Basilar invagination: a study based on 190 surgically treated patients. J Neurosurg 88: 962-968, 1998
T. Kaibara, R. J. Hurlbert, and G. R. Sutherland

13. Greenberg AD, Scoville WB, Davey LM: Transoral decompression of atlanto-axial dislocation due to odontoid hypoplasia. Report of two cases. J Neurosurg 28:266-269, 1968

14. Hadley MN, Spetzler RF, Sonntag VK: The transoral approach to the superior cervical spine. A review of 53 cases of extradural cervicomedullary compression. J Neurosurg 71:16-23, 1989

15. Hall WA, Liu H, Martin AJ, et al: Safety, efficacy, and functionality of high-field strength interventional magnetic resonance imaging for neurosurgery. Neurosurgery 46:632-41, 2000

16. Kaibara T, Saunders JK, Sutherland GR: Advances in mobile intraoperative magnetic resonance imaging. Neurosurgery 47: 131-137, 2000

17. Kaibara T, Saunders JK, Sutherland GR: Utility of a moveable 1.5 tesla intraoperative MR imaging system. Can J Neurol Sci 26:313-316, 1999

18. Menezes AH, VanGilder JC: Transoral-transpharyngeal approach to the anterior craniocervical junction. Ten-year experience with 72 patients. J Neurosurg 69:895-903, 1988

19. Menezes AH, VanGilder JC, Graf CJ, et al: Craniocervical abnormalities. A comprehensive surgical approach. J Neurosurg 53:444-455, 1980

20. Miller E, Crockard HA: Transoral transclival removal of anteriorly placed meningiomas at the foramen magnum. Neurosurgery 20:966-968, 1987

21. Mullan S, Naunton R, Hekmat-Panah J, et al: The use of an anterior approach to ventrally placed tumors in the foramen magnum and vertebral column. J Neurosurg 24:536-543, 1966

22. O'Laoire SA, Thomas DG: Transoral approach to the cervical spine: report of four cases. J Neurol Neurosurg Psychiatry 45:60-63, 1982

23. Pasztor E, Vajda J, Piffko P, et al: Transoral surgery for craniocervical space-occupying processes. J Neurosurg 60:276-281, 1984

24. Steinmeier R, Fahlbusch R, Ganslandt O, et al: Intraoperative magnetic resonance imaging with the magnetom open scanner: concepts, neurosurgical indications, and procedures: a preliminary report. Neurosurgery 43:739-747, 1998

25. Sutherland GR, Kaibara T, Louw D, et al: A mobile high-field magnetic resonance system for neurosurgery. J Neurosurg 91: 804-813, 1999

26. Tronnier VM, Wirtz CR, Knauth M, et al: Intraoperative diagnostic and interventional magnetic resonance imaging in neurosurgery. Neurosurgery 40:891-900, 1997

27. Wirtz CR, Tronnier VM, Bonsanto MM, et al: Image-guided neurosurgery with intraoperative MRI: update of frameless stereotaxy and radicality control. Stereotact Funct Neurosurg 68:39-43, 1997

Manuscript received December 18, 2000.

Accepted in final form January 19, 2001.

This work was supported by a grant from the Canadian Foundation for Innovation.

Address reprint requests to: Garnette R. Sutherland, M.D., The Seaman Family MR Research Center, Foothills Hospital, Division of Neurosurgery, University of Calgary, 1403 29th Street NW, Calgary, Alberta, T2N 2T9 Canada. email: garnette@ucalgary.ca. 